\title{
Erratum: Thermal quarkonium mass shift from Euclidean correlators [Phys. Rev. D 99, 094042 (2019)]
}

\author{
Alexander M. Eller, Jacopo Ghiglieriø, and Guy D. Moore
}

(Received 18 June 2020; published 14 August 2020)

DOI: 10.1103/PhysRevD.102.039901

A further investigation let us realize that the object we are calculating is not a thermal quarkonium mass shift, but the fluctuation counterpart to the momentum diffusion (dissipative) coefficient of a single heavy quark $\kappa$. We therefore would now change the title to "Thermal heavy quark self-energy from Euclidean correlators." In more detail, Eq. (2) would need revision. The object we consider precisely is

$$
\gamma_{\text {fund }}=\frac{g^{2}}{3 N_{\mathrm{c}}} \operatorname{Im} \int_{-\infty}^{\infty} d s\left\langle\operatorname{Tr}\left[\mathrm{P} U(\infty, s) E^{i}(s, \mathbf{0}) U(s, 0) E^{i}(0, \mathbf{0}) U(0,-\infty)\right]\right\rangle,
$$

where the chromoelectric fields $E$ and Wilson lines $U(a, b)$ are in the fundamental representation and $\mathrm{P}$ is the path-ordering symbol, indicating that fields are contour-ordered along the Wilson lines; this equals time ordering for the $E$ fields but not for the $U$ operators. The real part of the same quantity above is precisely $\kappa$ [1]. Hence, $\gamma_{\text {fund }}$ is related to the real part of the self-energy of a single heavy quark, though its precise physical interpretation is at the moment unclear. The real-time calculation in Ref. [2] used a Wilson line in the adjoint representation, i.e.,

$$
\gamma_{\mathrm{adj}}=\frac{g^{2}}{6 N_{\mathrm{c}}} \operatorname{Im} \int_{-\infty}^{\infty} d s\left\langle\mathrm{~T} E^{a, i}(s, \mathbf{0}) U(s, 0)_{a b} E^{b, i}(0, \mathbf{0})\right\rangle .
$$

It is $\gamma_{\text {adj }}$ which enters in the effective field theory description of heavy quarkonium at finite temperature.

The main message of the paper is that it is $\gamma_{\text {fund }}$ that can be analytically continued to the Euclidean correlation function. So, in Eq. (6), the following replacement should be done, $\gamma \rightarrow \gamma_{\text {fund }}$. A computational mistake was performed in the original version of the paper. Upon rectifying it, Eq. (11) becomes

$$
\gamma_{\text {fund }}^{\mathrm{LO}}=-\int_{0}^{\beta} d \tau G_{\mathrm{ENLO}}^{\mathrm{HQ}}(\tau)=-\frac{g^{4} C_{F}}{3} N_{f}\left(\tilde{\mathcal{I}}_{1}+4 \tilde{\mathcal{I}}_{2}\right)
$$

and Eq. (17)

$$
\gamma_{\text {fund }}^{\mathrm{LO}}=-2 \alpha_{\mathrm{s}}^{2} T^{3} \zeta(3) C_{F} N_{\mathrm{f}} .
$$

This is different from the result in Ref. [2], i.e.,

$$
\gamma_{\mathrm{adj}}^{\mathrm{LO}}=-2 \alpha_{\mathrm{s}}^{2} T^{3} \zeta(3) C_{F}\left(\frac{4}{3} N_{\mathrm{c}}+N_{\mathrm{f}}\right),
$$

since different Wilson lines were used. We have reproduced Eq. (3) by computing Eq. (1) in real time: we sketch that calculation at the end of this document in Appendix. Before we do so, we describe the changes to our conclusions: our updated main findings are that the different Wilson-line formulations $\gamma_{\text {fund }}$ and $\gamma_{\text {adj }}$ give rise to different results at the first nontrivial order in perturbation theory, and that it is $\gamma_{\text {fund }}$ which has a simple analytical continuation, Eq. (6) of the original paper. The difference between $\gamma_{\text {fund }}$ and $\gamma_{\text {adj }}$ may be understood physically as follows: $\gamma_{\text {fund }}$ is related to the propagation of a

Published by the American Physical Society under the terms of the Creative Commons Attribution 4.0 International license. Further distribution of this work must maintain attribution to the author(s) and the published articles title, journal citation, and DOI. 
single heavy quark, which can interact with the medium at any time, whereas $\gamma_{\text {adj }}$ describes a $Q \bar{Q}$ pair, which is a mediumblind singlet before (after) the first (last) $E$ field insertion. Indeed, our explicit evaluation, presented in Appendix, shows how the difference arises from the Wilson lines before/after the $E$ fields. We plan to return to the physical interpretation of $\gamma_{\text {fund }}$ in a follow-up publication, where we will also touch the issue of the Euclidean mapping for $\gamma_{\text {adj }}$ (of relevance for quarkonium) and that of $\kappa$, which we also expect to differ between the two Wilson-line formulations.

We thank Viljami Leino for uncovering the computational mistake that was performed in the original version of the paper.

\section{APPENDIX: DETAILS OF THE REAL-TIME COMPUTATION}

To our knowledge, there is no real-time determination of Eq. (1) in the literature; let us thus present it briefly. We find Coulomb gauge to be a good choice: in this gauge, the $A_{0} A_{0}$ retarded bare propagator is $G_{00}^{R}\left(q^{0}, q\right)=i / q^{2}$ and equals the advanced one. Thus, the spectral density vanishes, making the off-diagonal entries of the propagator matrix in the "12" formalism of real-time perturbation theory vanish. The lack of frequency dependence of the diagonal elements makes many diagrams vanish in dimensional regularization (DR). We label the real-time graphs as in Fig. 1 of the original paper, though the Wilson lines now start and end at $t=-\infty$. The only nonvanishing diagrams in this gauge are then (i), (j), and (k). Of these, (i) and (k) do not source any gluons from the Wilson lines: they thus contribute equally to $\gamma_{\text {adj }}$ and $\gamma_{\text {fund }}$, as the color trace gives the same result. In this gauge, any difference between the two can thus only arise from diagram (j) and its equivalent for $\gamma_{\text {adj. }}$. We thus show how the Coulomb gauge evaluation of the contribution of this diagram to Eqs. (2) and (1) yields the difference between $\gamma_{\text {adj }}$ and $\gamma_{\text {fund }}$, thus confirming the correctness of our analytical continuation, Eq. (6) of the original paper, to first nontrivial order in perturbation theory.

In the adjoint case, diagram (j) is shown in Fig. 1. It contributes to

$$
\begin{aligned}
\gamma_{\mathrm{adj}}^{(\mathrm{j})}= & -\frac{g^{4}}{3 N_{\mathrm{c}}} \operatorname{Im} \int_{0}^{\infty} d t \int_{0}^{t} d t^{\prime} \int_{Q} \int_{P} e^{i q^{0} t} e^{-i\left(p^{0}+q^{0}\right) t^{\prime}} \frac{i f^{a c b} f^{a b c}}{(\vec{p}+\vec{q})^{2}}\left[q^{0} p^{0}\left(q^{0}-p^{0}\right) G_{i k}^{11}(P) G_{k i}^{11}(Q)\right. \\
& \left.+2 i p^{0} \hat{q}^{i} \hat{q}^{j} G_{i j}^{11}(P)-2 i q^{0} \hat{p}^{i} \hat{p}^{j} G_{i j}^{11}(Q)\right],
\end{aligned}
$$

where we have rewritten the integral over negative and positive times of the contour-ordered operator as twice the positivetime integral of the forward Wightman operator. Thus, the three fields sourced by the operator, $E(t), A^{0}\left(t^{\prime}\right)$, and $E(0)$, are naturally time ordered and thus of type " 1 " in the 12 formalism of real-time perturbation theory. In Coulomb gauge, the $A^{0}\left(t^{\prime}\right)$ field can only connect to another $A^{0}$ field, which has furthermore to be of type 1 as well, due to the diagonal nature of the bare temporal propagator matrix. Hence, the three-gluon vertex has to be of type 1, so that the propagators of the transverse gluons have to be of type "11," i.e., time ordered. Indeed, the first line of Eq. (A1) is the contribution with two transverse gluons sourced by the two $E$ fields, as depicted in Fig. 1, while on the final line, they source one transverse and one temporal gluon. Finally, $\int_{P}=\int d^{D} P /(2 \pi)^{D}$ is the Minkowski $D$-dimensional integral.

In the fundamental case, one has instead the configurations shown in Fig. 2. They give

$$
\begin{aligned}
\gamma_{\text {fund }}^{(\mathrm{j})}= & -\frac{g^{4}}{6 N_{\mathrm{c}}} \operatorname{Im} \int_{0}^{\infty} d t\left(\int_{0}^{t} d t^{\prime}-\int_{-\infty}^{0} d t^{\prime}-\int_{t}^{\infty} d t^{\prime}\right) \int_{Q} \int_{P} e^{i q^{0} t} e^{-i\left(p^{0}+q^{0}\right) t^{\prime}} \frac{i f^{a c b} f^{a b c}}{(\vec{p}+\vec{q})^{2}} \\
& \times\left\{q^{0} p^{0}\left(q^{0}-p^{0}\right)\left[G_{i k}^{11}(P) G_{k i}^{11}(Q)+\epsilon\left(t^{\prime}\right) \epsilon\left(t-t^{\prime}\right) G_{i k}^{>}(P) G_{k i}^{>}(Q)\right]+2 i p^{0} \hat{q}^{i} \hat{q}^{j} G_{i j}^{11}(P)-2 i q^{0} \hat{p}^{i} \hat{p}^{j} G_{i j}^{11}(Q)\right\},
\end{aligned}
$$

where, as shown in the figure, there are now two possible 12 assignments for the fields sourced by the operator: the $E$ fields are always of type 1 , while the $A_{0}$ gluon is 1 if it comes from $U(0,-\infty)$ or $U(s, 0)$, "2" if from $U(-\infty, s)$. For reasons which will become clearer soon, we have rewritten this last Wilson line as $U(-\infty, s)=U(-\infty, \infty) U(\infty, s)$, with $U(-\infty, \infty)$ thus of type 2 and $U(\infty, s)$ of type 1 , hence the $\int_{t}^{\infty} d t^{\prime}$ contribution. ${ }^{2}$ The first term on the second line describes the diagrams with two transverse gluons, where we have used the definition $G^{>}=G^{21}$. For these diagrams, as shown in Fig. 2, we have two assignments contributing to each of the three $d t^{\prime}$ integrations. The relative sign between the two, encoded in the sign

\footnotetext{
${ }^{1}$ Reference [2] obtained Eq. (4) in the temporal axial gauge $A_{0}=0$. We also checked that the sum of diagrams (i), (j), and (k) in the expansion of $\gamma_{\text {adj }}$ in Coulomb gauge reproduces Eq. (4).

${ }^{2}$ In a covariant gauge, the contribution of $\int_{t}^{\infty} d t^{\prime}$ vanishes, as expected from the unitarity of the Wilson lines. In Coulomb gauge, one needs anyway to consider $U(-\infty, s)$ as $U\left(-\infty, s+\delta^{+}\right) U\left(\delta^{+}, s\right)$, with $\delta^{+}$arbitrarily small and positive. This avoids the appearance of ill-defined $\theta(0)$ contributions arising from the time integrations of the bare temporal propagators, which are instantaneous in time.
} 


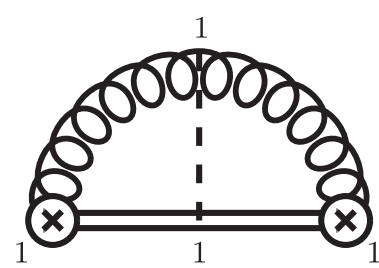

FIG. 1. Diagram (j) for $\gamma_{\text {adj }}^{(\mathrm{j})}$. The vertices with the cross are the $E$ fields, the double line is the adjoint Wilson line, curly lines are transverse gluons, and the dashed line a temporal gluon. The diagram where one of the $E$ fields sources a temporal gluon is not shown explicitly. The 1 labels the 12 assignments of the fields.

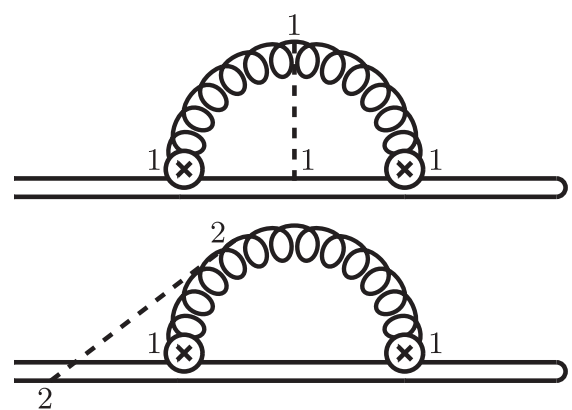

FIG. 2. Diagrams (j) for $\gamma_{\text {fund }}^{(\mathrm{j})}$. The graphical notation is the same as in Fig. 1, except that the solid line is now the Wilson line stretching forward in time from negative to positive infinity passing both $E$ fields (upper contour), to then turn back and return to $-\infty$ (lower contour). We show two of the six possibilities for the temporal gluon, which can connect as a 1 (2) field to the upper (lower) contour before, between or after the $E$ fields. We do not show the case where the $E$ fields source one transverse and one temporal gluon; there the lower contour does not contribute.

functions $\epsilon\left(t^{\prime}\right) \epsilon\left(t-t^{\prime}\right)$, arises from the combination of a minus sign from the different color ordering -in the $0<t^{\prime}<t$ region - together with another minus sign from the different direction of the Wilson lines. The final two terms on the second line encode the contribution of graphs with a single transverse gluon, for which the lower 2 contour does not contribute. The overall factor of $1 / 2$ in front of Eq. (A2) with respect to Eq. (A1) arises from color tracing in the different cases.

If we take the difference between Eqs. (A2) and (A1), we obtain $\Delta \gamma \equiv \gamma_{\text {fund }}-\gamma_{\text {adj }}$. It reads

$$
\begin{aligned}
\Delta \gamma= & -\frac{g^{4} C_{F} N_{\mathrm{c}}}{3} \operatorname{Im} \int_{0}^{\infty} d t \int_{-\infty}^{\infty} d t^{\prime} \int_{Q} \int_{P} \frac{i e^{i q^{0} t} e^{-i\left(p^{0}+q^{0}\right) t^{\prime}}}{(\vec{p}+\vec{q})^{2}} \\
& \times\left\{q^{0} p^{0}\left(q^{0}-p^{0}\right)\left[G_{i k}^{11}(P) G_{k i}^{11}(Q)-G_{i k}^{>}(P) G_{k i}^{>}(Q)\right]+2 i p^{0} \hat{q}^{i} \hat{q}^{j} G_{i j}^{11}(P)-2 i q^{0} \hat{p}^{i} \hat{p}^{j} G_{i j}^{11}(Q)\right\},
\end{aligned}
$$

so that the structure of the time integrations simplifies greatly, hence our choice of introducing the $\int_{t}^{\infty} d t^{\prime}$ contribution. Upon using $G_{i j}(P)=\left(\delta_{i j}-\hat{p}_{i} \hat{p}_{j}\right) G_{T}(P)$, we find

$$
\begin{aligned}
\Delta \gamma= & -\frac{2 g^{4} C_{F} N_{\mathrm{c}}}{3} \operatorname{Im} \int_{Q} \int_{P} \frac{2 \pi \delta\left(q^{0}+p^{0}\right)}{(\vec{p}+\vec{q})^{2}} \times\left\{p_{0}^{2}\left[G_{T}^{11}(P) G_{T}^{11}(Q)-G_{T}^{>}(P) G_{T}^{>}(Q)\right]\left[d-2+(\hat{p} \cdot \hat{q})^{2}\right]\right. \\
& \left.+i\left[G_{T}^{11}(P)+G_{T}^{11}(Q)\right]\left[1-(\hat{p} \cdot \hat{q})^{2}\right]\right\} .
\end{aligned}
$$

$G_{T}^{>}(Q)=\left(\theta\left(q^{0}\right)+n_{\mathrm{B}}\left(\left|q^{0}\right|\right)\right) 2 \pi \delta\left(Q^{2}\right)$ is purely real and thus does not contribute to $\Delta \gamma$. $G_{T}^{11}(Q)=i \mathbf{P} 1 /\left(q_{0}^{2}-q^{2}\right)+$ $\left(1 / 2+n_{\mathrm{B}}\left(\left|q^{0}\right|\right)\right) 2 \pi \delta\left(Q^{2}\right)$ has both real and imaginary parts, with $\mathbf{P}$ a principal-value prescription and $n_{\mathrm{B}}$ the Bose-Einstein distribution, so that

$$
\Delta \gamma=-4 \frac{g^{4} C_{F} N_{\mathrm{c}}}{3} \int_{Q} \int_{p} \frac{2 \pi \delta\left(Q^{2}\right)}{(\vec{p}+\vec{q})^{2}}\left[\frac{1}{2}+n_{\mathrm{B}}\left(\left|q^{0}\right|\right)\right]\left\{\mathbf{P} \frac{q_{0}^{2}}{q_{0}^{2}-p^{2}}\left[d-2+(\hat{p} \cdot \hat{q})^{2}\right]+1-(\hat{p} \cdot \hat{q})^{2}\right\}=\frac{8}{3} \alpha_{\mathrm{s}}^{2} C_{F} N_{\mathrm{c}} \zeta(3) T^{3},
$$


where we have also used the $p \leftrightarrow q$ symmetry of the integrand. The final integration has been carried out in DR, showing that Eq. (A5) is equal to the difference between Eqs. (3) and (4), as we set out to prove.

[1] J. Casalderrey-Solana and D. Teaney, Phys. Rev. D 74, 085012 (2006).

[2] N. Brambilla, J. Ghiglieri, A. Vairo, and P. Petreczky, Phys. Rev. D 78, 014017 (2008). 